NOVA

University of Newcastle Research Online

nova.newcastle.edu.au

Best, D. J.; Rayner, J. C. W. "Chi-squared components for tests of fit and improved models for the grouped exponential distribution" Computational Statistics and Data Analysis Vol. 51, Issue 8, p. 3946-3954 (2007)

Available from: http://dx.doi.org/10.1016/i.csda.2006.03.014

Accessed from: http://hdl.handle.net/1959.13/926789 


\title{
Chi-Squared Components for Tests of Fit and Improved Models for the Grouped Exponential Distribution
}

\author{
D.J. Best ${ }^{*}$ and J. C. W. Rayner
}

\begin{abstract}
We consider testing for an exponential distribution with unspecified rate parameter when it is only possible to observe the counts in groups with boundaries specified before sighting the data. On the basis of a size and power study we recommend that tests of fit for the exponential distribution be based on the Anderson-Darling statistic and the SW2 statistic recommended by Gulati and Neus (2001). We also suggest that inference based on one of these be complemented by examination of the components of the $X^{2}$ statistic. We illustrate how to use these components to give improved models.
\end{abstract}

Key Words: Improved grouped exponential models; Method of moments estimation; Size and power study.

\section{INTRODUCTION}

Some rivers have gravel-bed channels that are scoured during floods. Measuring devices for the depth of scouring can only give results within certain grouping intervals. Spinelli (2001) gives the results in Table 1 for 39 floods of a river at a given location.

Table 1. Scour depth values (in $\mathrm{cm}$ ).

\begin{tabular}{|c|c|c|c|c|c|c|}
\hline & $0-2$ & $2-6$ & $6-10$ & $10-14$ & $14-18$ & $>18$ \\
\hline Frequency & 21 & 9 & 5 & 2 & 1 & 1 \\
\hline Cell expectations $\left(n p_{j}\right)$ & 15.9 & 15.0 & 5.2 & 1.8 & 0.6 & 0.3 \\
\hline Cell expectations $\left(n p_{j}^{*}\right)$ & 19.6 & 11.9 & 3.4 & 1.8 & 1.2 & 1.1 \\
\hline
\end{tabular}

Do the data follow a grouped exponential distribution? Suppose a random sample of $n$ observations is available but all that is known about these observations is into which of $K$ mutually exclusive predetermined groups they fall. The $K$ groups have boundaries $k_{1}, k_{2}, \ldots$, $k_{K-1}$ specified before sighting the data. We also take $k_{0}=0$ and $k_{K}=\infty$. The null hypothesis to be tested is that the data comes from an exponential distribution with probability density function

$$
f(y ; \theta)=\theta \exp (-\theta y) \text { for } y>0 \text {, zero otherwise, in which } \theta>0 \text {. }
$$

\footnotetext{
*Correspondence: D.J. Best, School of Mathematical and Physical Sciences, University of Newcastle, Callaghan, NSW 2308, Australia; Fax: 61-2-49216898; E-mail:

John.Best@newcastle.edu.au
} 
For $j=1,2, \ldots, K$ the probability of an observation in group $j$ is $p_{j}=\exp \left(-\theta k_{j-1}\right)-\exp \left(-\theta k_{j}\right)$. Again for $j=1,2, \ldots, K$ let $N_{j}$ be the number of observations that fall into the $j$ th group and let $x_{j}=\left(k_{j}+k_{j-1}\right) / 2$ for $j=1,2, \ldots, K-1$ while $x_{K}=k_{K-1}+\left(k_{K-1}-k_{K-2}\right) / 2=\left(3 k_{K-1}-k_{K-2}\right) / 2$. The sample mean of the grouped data is given by $\bar{X}=\sum_{j=1}^{K} N_{j} x_{j} / n$ and the grouped population mean by $\mu=\sum_{j=1}^{K} p_{j} x_{j}$. The method of moments estimator (MME) of $\theta$, say $\hat{\theta}$, is obtained by iteratively solving $\mu=\bar{X}$ for $\theta$. If $\theta_{0}=1 / \bar{X}$ is taken as a first estimate of $\hat{\theta}$ then, using Newton's method, a better estimate is

$$
\theta_{1}=\theta_{0}-(\mu-\bar{x}) / \sum_{j=1}^{K}\left\{k_{j} \exp \left(-k_{j} \theta\right)-k_{j-1} \exp \left(-k_{j-1} \theta\right)\right\} x_{j}
$$

Repeat this process to find $\theta_{2}, \theta_{3}, \ldots$ until convergence is reached. Spinelli (2001) describes how to find the maximum likelihood estimator (MLE) of $\theta$ while Thas et al. (2006) discuss theory that supports use of the MME. The generalised score test is used to construct an omnibus test statistic which is a quadratic form in the components with the unknown nuisance parameters estimated by equating the initial components (defined in the next section) to zero. Their model involves orthonormal functions, and if these are the orthonormal polynomials then the method of estimation is MME. For the scenario here only one parameter is estimated and the omnibus test statistic is $X^{2}$ with $K$ - 2 degrees of freedom.

Once $\theta$ has been estimated it is straightforward to apply this test of fit for the grouped exponential distribution. For the Table 1 data $1 / \hat{\theta}=3.81$, which is very similar to the MLE value of 3.82 given in Spinelli (2001). If no pooling is done for the Table 1 data then $X^{2}=$ $\sum_{j=1}^{K}\left(n_{j}-n p_{j}\right)^{2} /\left(n p_{j}\right)=5.48$ on four degrees of freedom. The corresponding p-value is 0.24 using the $\chi_{4}^{2}$ approximation to $X^{2}$ and 0.21 using a parametric bootstrap based on 1,000 simulations. It appears the data are consistent with the grouped exponential distribution. Table 1 also gives the cell expectations, $E_{j}=n p_{j}$, used in calculating $X^{2}$.

\section{COMPONENTS OF THE CHI-SQUARED STATISTIC}

A more thorough examination of the null grouped exponential hypothesis can be made by using the components of the $X^{2}$ statistic, successfully exploited in Best and Rayner (2003, 2005, 2006) to test for the Geometric, Poisson and Binomial distributions. It was thus thought worthwhile to examine this components of $X^{2}$ approach to test for the grouped exponential distribution, recently considered by Spinelli (2001) and Gulati and Neus (2001). Extra complications in the grouped exponential case are that the MME and MLE no longer coincide and iterative methods are needed to find both of these estimators.

If a discrete random variable $Y$ with $P\left(Y=y_{j}\right)=p_{j}$ for $j=1, \ldots, K$ has mean $\mu$ and central moments $\mu_{r}=\sum_{j=1}^{K}\left(y_{j}-\mu\right)^{r} p_{j}, r=2,3, \ldots, K$, then the initial orthonormal polynomials are

$$
\begin{gathered}
g_{0}\left(y_{j}\right)=1 \text { for all } x, g_{1}\left(y_{j}\right)=\left(y_{j}-\mu\right) / \sqrt{\mu_{2}}, \\
g_{2}\left(y_{j}\right)=\left\{\left(y_{j}-\mu\right)^{2}-\mu_{3}\left(y_{j}-\mu\right) / \mu_{2}-\mu_{2}\right\} / \sqrt{\mu_{4}-\mu_{3}^{2} / \mu_{2}-\mu_{2}^{2}} \text { and }
\end{gathered}
$$




$$
g_{3}\left(y_{j}\right)=\frac{\left(y_{j}-\mu\right)^{3}-a\left(y_{j}-\mu\right)^{2}-b\left(y_{j}-\mu\right)-c}{\sqrt{\mu_{6}-2 a \mu_{5}+\left(a^{2}-2 b\right) \mu_{4}+2(a b-c) \mu_{3}+\left(b^{2}+2 a c\right) \mu_{2}+c^{2}}}
$$

in which

$$
\begin{gathered}
a=\left(\mu_{5}-\mu_{3} \mu_{4} / \mu_{2}-\mu_{2} \mu_{3}\right) / d, b=\left(\mu_{4}^{2} / \mu_{2}-\mu_{2} \mu_{4}-\mu_{3} \mu_{5} / \mu_{2}+\mu_{3}^{2}\right) / d, \\
c=\left(2 \mu_{3} \mu_{4}-\mu_{3}^{3} / \mu_{2}-\mu_{2} \mu_{5}\right) / d \text { and } d=\mu_{4}-\mu_{3}^{2} / \mu_{2}-\mu_{2}^{2} .
\end{gathered}
$$

Further orthonormal polynomials may be defined using the recurrence relations in Emerson (1968). Now the $r$ th component of $X^{2}$ is defined as

$$
V_{r}=\sum_{j=1}^{K} N_{j} g_{r}\left(y_{j}\right) / \sqrt{n} \text { for } r=1,2, \ldots, K-1
$$

and, as in Lancaster (1953),

$$
X^{2}=V_{1}^{2}+\ldots+V_{K-1}^{2}
$$

For the data in Table $1 V_{1}=0$ as we have estimated $\theta$ by the MME, and $V_{2}^{2}=4.21$. As $X^{2}=$ 5.48 none of the other components $V_{3}, V_{4}$ or $V_{5}$ can be very large, so only $V_{2}$ is important. Both the $\chi_{1}^{2}$ and the parametric bootstrap p-values for $V_{2}^{2}$ are 0.03 , suggesting possible differences in dispersion from the hypothesised distribution. The $V_{2}^{2}$ statistic provides a more sensitive directed test of the null hypothesis than $X^{2}$ for the Table 1 data. In general a large $V_{r}^{2}$ suggests deviations from the null hypothesis in moments up to the $r$ th moment. Rayner et al. (1995) discuss this point. It is possible that $X^{2}$ is nonsignificant while one of the components is significant, and in this case we say that the significant component has been 'diluted' by other nonsignificant components.

Since $V_{2}^{2}$ is significantly large an improved model for the Table 1 data is given by

$$
p_{j}^{*}=C p_{j}\left\{1+V_{2} g_{2}\left(x_{j}\right) / \sqrt{ } n\right\} \text { if } p_{j}^{*}>0 \text { and zero otherwise for } j=1,2, \ldots, 6 \text {, }
$$

in which $C$ is a normalising constant introduced to ensure that $\sum_{j} p_{j}^{*}=1$. Note that if all $p_{j}^{*}$ $>0$ then $C=1$. Similar models were discussed in Stuart and Ord (1994, section 6.24) and Rayner and Best (1989, p.97). It is well known that a problem with the Charlier and GramCharlier models is that they can produce negative probabilities. In such cases we take $p_{j}^{*}=0$. The final row of Table 1 gives the cell expectation $n p_{j}^{*}$ so that the observations can be compared with the expectations from both the $\left\{p_{j}\right\}$ and the $\left\{p_{j}^{*}\right\}$ models. The latter are clearly closer to the observed counts.

For the reader who wishes to calculate $V_{2}$ we now give some details for the scour depth data, for which $\hat{\theta}=0.26278$. If there was no data grouping we would have $\hat{\mu}=1 / \hat{\theta}=$ 3.806. However, because of the data grouping we find $\left\{p_{j}\right\}=\{0.4088,0.3846,0.1344$, 0.0470, 0.0164, 0.0088\} and $\hat{\mu}=\sum_{j=1}^{6} x_{j} p_{j}=4.0256, \hat{\mu}_{2}=\sum_{j=1}^{6}\left(x_{j}-\mu\right)^{2} p_{j}=13.4613, \hat{\mu}_{3}$ $=\sum_{j=1}^{6}\left(x_{j}-\mu\right)^{3} p_{j}=85.1270$ and $\hat{\mu}_{4}=\sum_{j=1}^{6}\left(x_{j}-\mu\right)^{4} p_{j}=1170.2790$. As above $g_{2}\left(x_{j}\right)=$ $0.0471\left\{\left(x_{j}-\hat{\mu}\right)^{2}-\left(x_{j}-\hat{\mu}\right) \hat{\mu}_{3} / \hat{\mu}_{2}-\hat{\mu}_{2}\right\}$. We obtain $g_{2}(1)=0.6984, g_{2}(2)=-0.6264, g_{2}(3)=-$ 
1.0739, $g_{2}(4)=0.0141, g_{2}(5)=2.5529$ and $g_{2}(6)=6.6272$. Thus $V_{2}=\sum_{j=1}^{6} n_{j} g_{2}\left(x_{j}\right) / \sqrt{39}=$ $(21 \times 0.6984-9 \times 0.6264-5 \times 1.0739+\ldots+1 \times 6.6272) / \sqrt{ } 39=2.0514$.

Alternatively let $\left\{w_{j}\right\}=\left\{\left(n_{1}-E_{1}\right) / E_{1}, \ldots,\left(n_{6}-E_{6}\right) / E_{6}\right\}$ where $E_{j}=39 p_{j}$, and assign $\left\{z_{j}\right\}=\left\{x_{j}-\hat{\mu}\right\}$. We have made this assignment to avoid rounding error in the following regression analysis. The sum of squares for the weighted regression of $T$ on $z$ is 0.000 when the weights are $E_{j}, j=1, \ldots, 6$. This sum of squares was expected as $V_{1}$ is close to zero due to the estimation of $\theta$. Using the same weights the sum of squares for the weighted regression of $T$ on $z$ and $z^{2}$ is 4.21 . This is $V_{2}^{2}$.

If the null hypothesis is rejected improved models can be constructed by making adjustments like that above, including terms in the hypothesised cell probabilities corresponding to the components with sufficiently small p-values.

\section{POWER COMPARISONS}

Powers for the tests based on the statistics $X^{2}, V_{2}^{2}$ and $V_{3}^{2}$ were calculated and compared with powers for the Anderson-Darling $A^{2}$ test studied by Spinelli (2001) and the tests based on the SW1 and SW2 statistics proposed by Gulati and Neus (2001). Tables 2 and 3 give comparisons, for $n=100$, test size $\alpha=0.05$, and for when the null hypothesis ungrouped data have means of 2.0 and 4/3. The alternatives considered involve commonly used skewed alternatives to the exponential and were those used in Spinelli (2001). They are based on a gamma $(\alpha, \beta)$ distribution with density proportional to $y^{a-1} e^{-\beta y}$, a Weibull $(\alpha, \beta)$ distribution with cumulative distribution function $1-\exp (-y / \beta)^{\alpha}$, and a lognormal $(\alpha, \beta)$ distribution with associated random variable $Y$ such that $(\log Y-\alpha) / \beta$ has a standard normal distribution. Random values from these distributions were obtained using the IMSL (1995) software package.

Spinelli (2001) gives three groups of null distributions. Our power comparisons use the second and third of these groups as his first group classifies most of the data into only three classes, and this does not allow us to examine $V_{3}^{2}$. Further, Spinelli (2001) sets up his null distributions for his second and third groups of null distributions with 26 classes and an infinite number of classes respectively. We suggest that in practice it is sensible to use far fewer classes. We have used $K=6$ in our Tables 2 and 3. We also assume that in practice it would not be possible to choose $K$ : it would be given. Thus in Table $2 \theta=0.5$ and the cut points are $k_{1}=0.5, k_{2}=1.5, k_{2}=2.5, k_{4}=3.5, k_{5}=4.5$, while in Table $3 \theta=0.75$ and the cut points are $k_{i}=i, i=1, \ldots, 5$. We denote the former specification as $\mathrm{A}$, and that for Table 3 as B.

Table 2. Powers for null specification A; test size 0.05 and $n=100$.

\begin{tabular}{|lccccccc|}
\hline Alternative & $X^{2}$ & $V_{2}^{2}$ & $A^{2}$ & $V_{3}^{2}$ & $S W 1$ & $S W 2$ & $\sigma^{2}$ \\
\hline Gamma (.75, .375) & 0.25 & 0.36 & 0.36 & 0.07 & 0.34 & 0.40 & 5.33 \\
Gamma (1.5, 0.75) & 0.48 & 0.63 & 0.65 & 0.09 & 0.62 & 0.67 & 2.67 \\
Exponential (0.5) & 0.06 & 0.04 & 0.06 & 0.05 & 0.05 & 0.06 & 4.00 \\
Weibull $(0.85,1.838)$ & 0.20 & 0.32 & 0.30 & 0.06 & 0.28 & 0.34 & 5.58 \\
Weibull $(1.2,2.1126)$ & 0.29 & 0.44 & 0.41 & 0.05 & 0.43 & 0.44 & 2.80 \\
Lognormal (0.336, 0.845) & 0.82 & 0.61 & 0.92 & 0.63 & 0.79 & 0.88 & 4.17 \\
Lognormal (0.405, 0.759) & 0.98 & 0.93 & 0.99 & 0.74 & 0.98 & 0.99 & 3.12 \\
Lognormal (0.182, 1.011) & 0.27 & 0.06 & 0.27 & 0.39 & 0.19 & 0.23 & 7.12 \\
\hline
\end{tabular}


Table 3. Powers for null specification B; test size 0.05 and $n=100$.

\begin{tabular}{|lccccccc|}
\hline Alternative & $X^{2}$ & $V_{2}^{2}$ & $A^{2}$ & $V_{3}^{2}$ & $S W 1$ & $S W 2$ & $\sigma^{2}$ \\
\hline Gamma (0.55, 0.412) & 0.53 & 0.69 & 0.67 & 0.07 & 0.64 & 0.70 & 3.24 \\
Gamma (1.5, 1.125) & 0.27 & 0.41 & 0.47 & 0.10 & 0.39 & 0.46 & 1.19 \\
Exponential (0.75) & 0.06 & 0.05 & 0.05 & 0.06 & 0.05 & 0.05 & 1.78 \\
Weibull (0.75, 1.12) & 0.43 & 0.56 & 0.51 & 0.07 & 0.54 & 0.55 & 3.25 \\
Weibull (1.4, 1.463) & 0.64 & 0.84 & 0.82 & 0.19 & 0.82 & 0.84 & 0.93 \\
Lognormal (-0.069, 0.845) & 0.12 & 0.07 & 0.13 & 0.17 & 0.10 & 0.12 & 1.85 \\
Lognormal (0.065, 0.668) & 0.62 & 0.58 & 0.80 & 0.47 & 0.64 & 0.77 & 1.00 \\
Lognormal (-0.223, 1.011) & 0.24 & 0.33 & 0.19 & 0.13 & 0.32 & 0.25 & 3.17 \\
\hline
\end{tabular}

Powers were calculated using a parametric bootstrap procedure similar to that given in Gulati and Neus (2001). A grouped random sample of size $n=100$ was produced for each alternative and $\hat{\theta}$ calculated. The test statistics were calculated and then 1,000 grouped exponential samples with parameter $\hat{\theta}$ were generated. The proportion of these 1,000 samples with the statistic values greater than the value from the original sample was found. This is called the bootstrap p-value. This procedure was repeated 1,000 times. The power of each of the tests was then taken as the proportion of the bootstrapped p-values less than the test size, 0.05. Some checks with 10,000 bootstrap samples and 10,000 repetitions made little difference in the estimated powers.

From Tables 2 and 3 we see that the tests based on $V_{2}^{2}, A^{2}$, SW1 and SW2 largely all perform as well as each other. An exception is when the variance under the alternative is close to the variance under the null: see Lognormal $(0.336,0.845)$ and Lognormal $(-0.069,0.845)$ alternatives, when $V_{2}^{2}$ has poor power. In such cases the power of $X^{2}$ seems reasonable. The variance of the ungrouped alternative is given in the column headed $\sigma^{2}$.

Further examination of Tables 2 and 3 indicates that the test based on $V_{3}^{2}$ does not have much power except for the Lognormal alternatives. Possibly this indicates that most of the data are concentrated in the first few classes. Further, as noted previously by Gulati and Neus (2001), the $S W 2$ test seems to be almost always more powerful that the $S W 1$ test.

On the basis of our power calculations we suggest tests based $A^{2}$ and $S W 2$ be used as formal tests of exponentiality for grouped data. As an informal check of the data we also suggest calculating $V_{2}^{2}, V_{3}^{2}$ and $X^{2}$ and approximate p-values based on $\chi_{1}^{2}$ and $\chi_{K-2}^{2}$ as appropriate. These p-values may then to be used to indicate possible deviations between the data and the hypothesised exponential model in dispersion and other moments. Some simulations not reported here indicate these approximations work well when the MME and MLE are close. Moreover the components of $X^{2}$ can be used to give an improvement on the exponential model.

\section{THE NON-CENTRAL CHI-SQUARED POWER APPROXIMATION}

In previous studies, such as Best and Rayner (2003, 2005, 2006), we have found that the powers for $X^{2}$ and $V_{2}^{2}$ can be approximated, with variable success, by the non-central chisquared distribution. In this section we briefly examine this approximation for the grouped data exponential distribution. See Tables 4 and 5.

When all class expectations are greater than one and mostly greater than five, the noncentral chi-squared approximation is good enough to be useful, for example, in calculating the approximate sample size needed to achieve a specified power. However when the class expectations do not meet this criterion, the non-central chi-squared approximation cannot be recommended. For example see the Weibull $(1.4,1.463)$ case in Table 5. 
Table 4. Bootstrap (B) and non-central (NC) chi-squared powers for null specification A; test size 0.05 and $n=100$.

\begin{tabular}{|lcccc|}
\hline Alternative & $X^{2}(\mathrm{~B})$ & $X^{2}(\mathrm{NC})$ & $V_{2}^{2}(\mathrm{~B})$ & $V_{2}^{2}(\mathrm{NC})$ \\
\hline Gamma $(.75, .375)$ & 0.25 & 0.23 & 0.36 & 0.35 \\
Gamma (1.5, 0.75) & 0.48 & 0.57 & 0.63 & 0.61 \\
Exponential $(0.5)$ & 0.06 & 0.05 & 0.04 & 0.05 \\
Weibull $(0.85,1.838)$ & 0.20 & 0.20 & 0.32 & 0.31 \\
Weibull $(1.2,2.1126)$ & 0.29 & 0.30 & 0.44 & 0.41 \\
Lognormal $(0.336,0.845)$ & 0.82 & 0.90 & 0.61 & 0.58 \\
Lognormal (0.405, 0.759) & 0.98 & 0.99 & 0.93 & 0.89 \\
Lognormal (0.182, 1.011) & 0.27 & 0.31 & 0.06 & 0.06 \\
\hline
\end{tabular}

Table 5. Bootstrap (B) and non-central (NC) chi-squared powers for null specification B; test size 0.05 and $n=100$.

\begin{tabular}{|lcccc|}
\hline Alternative & $X^{2}(\mathrm{~B})$ & $X^{2}(\mathrm{NC})$ & $V_{2}^{2}(\mathrm{~B})$ & $V_{2}^{2}(\mathrm{NC})$ \\
\hline Gamma $(0.55,0.412)$ & 0.53 & 0.48 & 0.69 & 0.71 \\
Gamma $(1.5,1.125)$ & 0.27 & 0.31 & 0.41 & 0.36 \\
Exponential $(0.75)$ & 0.06 & 0.05 & 0.05 & 0.05 \\
Weibull $(0.75,1.12)$ & 0.43 & 0.41 & 0.56 & 0.64 \\
Weibull $(1.4,1.463)$ & 0.64 & 0.93 & 0.84 & 0.75 \\
Lognormal $(-0.069,0.845)$ & 0.12 & 0.14 & 0.07 & 0.05 \\
Lognormal (0.065, 0.668) & 0.62 & 0.63 & 0.58 & 0.49 \\
Lognormal $(-0.223,1.011)$ & 0.24 & 0.24 & 0.33 & 0.28 \\
\hline
\end{tabular}

Another reason why the $V_{2}^{2}$ powers are not exactly non-central chi-squared distributed even for large samples, is that $\operatorname{var}\left(V_{2}\right)$ is not asymptotically one as it was in the Geometric, Poisson and Binomial cases studied in Best and Rayner (2003, 2005, 2006). However for data sets we have considered, MME and MLE are often close for grouped exponential data and so the powers for $V_{2}^{2}$ (B) and $V_{2}^{2}$ (NC) in Table 5 may represent common situations. Thas et al. (2006) give general theory for when $\operatorname{var}\left(V_{r}\right)$ is one.

\section{BUS-MOTOR FAILURES}

Spinelli (2001) considered the scour depth data from a river bed that we presented in Table 1. He calculated $A^{2}$ for these data and found a value of 1.2215 with p-value 0.04 . In section 2 we found the data differed in dispersion from what could be expected for grouped exponential data: $V_{2}^{2}=4.21$ with p-value 0.03 based on a $\chi_{1}^{2}$ approximation. We also gave and, in Table 1, illustrated, an improved model: $p_{j}^{*}=p_{j}\left\{1+V_{2} g_{2}\left(x_{j}\right) / \downarrow_{n}\right\}$ for $j=1,2, \ldots, 6$. We now further illustrate our approach using the bus-motor failure data given in Davis (1952) and considered recently by Mudholkar et al. (1995) and Gulati and Neus (2003).

The bus-motor failures concern an initial fleet of 191 buses. The data are the number of miles in thousands for the first, second, ..., fifth motor failure. Davis (1952) gives further details. Mudholkar et al. (1995) show that the data may be well modelled by an exponentiated Weibull distribution, while Gulati and Neus (2003) use SW2 to show that the first, second and fifth data sets are not well fitted by a grouped exponential model. 
Table 6. First bus-motor failures.

\begin{tabular}{|c|c|c|c|c|}
\hline & & \multicolumn{3}{|c|}{$\begin{array}{l}\text { Expected } \\
\text { frequency }\end{array}$} \\
\hline $\begin{array}{l}\text { Class Interval } \\
\text { (1,000 miles) }\end{array}$ & $\begin{array}{l}\text { Observed } \\
\text { frequency }\end{array}$ & Exponential & $\begin{array}{l}\text { Exponentiated } \\
\text { Weibull }\end{array}$ & $n p_{j}^{*}$ \\
\hline $0-20$ & 6 & 26.5 & 3.9 & 7.9 \\
\hline $20-40$ & 11 & 22.9 & 11.8 & 6.4 \\
\hline $40-60$ & 16 & 19.7 & 19.7 & 16.6 \\
\hline $60-80$ & 25 & 16.9 & 27.5 & 28.7 \\
\hline $80-100$ & 34 & 14.6 & 34.5 & 37.1 \\
\hline $100-120$ & 46 & 12.6 & 38.4 & 38.8 \\
\hline $120-140$ & 33 & 10.8 & 33.8 & 33.0 \\
\hline $140-160$ & 16 & 9.3 & 18.0 & 19.9 \\
\hline \multirow[t]{2}{*}{$>160$} & 4 & 57.7 & 3.4 & 2.6 \\
\hline & & $\begin{array}{c}\hat{\theta}=0.00748, \\
X^{2}=241.70, \mathrm{df}=7, \\
\text { p-value } 0.00 \\
V_{2}^{2}=169.2, \\
\text { p-value } 0.00 \\
V_{3}^{2}=64.2, P=0.00\end{array}$ & $\begin{array}{c}X^{2}=2.7, \mathrm{df}=3, \\
\text { p-value } 0.45\end{array}$ & $\begin{array}{c}X^{2}=7.35, \mathrm{df}=5, \\
\text { p-value } 0.20\end{array}$ \\
\hline
\end{tabular}

Table 7. Second bus-motor failures.

\begin{tabular}{|c|c|c|c|c|}
\hline & & \multicolumn{3}{|c|}{$\begin{array}{c}\text { Expected } \\
\text { frequency }\end{array}$} \\
\hline $\begin{array}{c}\text { Class Interval } \\
(1,000 \text { miles })\end{array}$ & $\begin{array}{c}\text { Observed } \\
\text { frequency }\end{array}$ & Exponential & $\begin{array}{c}\text { Exponentiated } \\
\text { Weibull }\end{array}$ & $n p_{j}^{*}$ \\
\hline $0-20$ & 19 & 21.5 & 16.8 & 20.5 \\
$20-40$ & 13 & 17.0 & 15.8 & 9.8 \\
$40-60$ & 13 & 13.5 & 15.4 & 13.0 \\
$60-80$ & 15 & 10.7 & 15.2 & 17.4 \\
$80-100$ & 15 & 8.5 & 15.0 & 17.7 \\
$100-120$ & 18 & 6.8 & 14.6 & 12.6 \\
$>120$ & 11 & 25.9 & 11.1 & 13.0 \\
& & $\hat{\theta}=0.01156$, & & $X^{2}=4.47, \mathrm{df}=3$, \\
& & $X^{2}=35.25, \mathrm{df}=5$, & $X^{2}=1.95, \mathrm{df}=3$, & $\mathrm{p}$-value 0.21 \\
& & $\mathrm{p}$-value 0.00 & p-value 0.58 & \\
& & $V_{2}^{2}=10.82$, & & \\
& & $\mathrm{p}$-value 0.00 & & \\
& & $V_{3}^{2}=17.91$, & & \\
& & $\mathrm{p}$-value 0.00 & & \\
& &
\end{tabular}

In Tables 6 to 10 we calculate our $V_{2}$ and $V_{3}$ statistics as well as comparing the improved model

$$
p_{j}^{*}=p_{j}\left\{1+V_{2} g_{2}\left(x_{j}\right) / \sqrt{ } n+V_{3} g_{3}\left(x_{j}\right) / \sqrt{ } n\right\} \text { if } p_{j}^{*}>0 \text { and zero otherwise for all } j
$$


Table 8. Third bus-motor failures.

\begin{tabular}{|c|c|c|c|c|}
\hline & & \multicolumn{3}{|c|}{$\begin{array}{c}\text { Expected } \\
\text { frequency }\end{array}$} \\
\hline $\begin{array}{c}\text { Class Interval } \\
(1,000 \text { miles })\end{array}$ & $\begin{array}{c}\text { Observed } \\
\text { frequency }\end{array}$ & Exponential & $\begin{array}{c}\text { Exponentiated } \\
\text { Weibull }\end{array}$ & $n p_{j}^{*}$ \\
\hline $0-20$ & 27 & 27.5 & 24.6 & 26.7 \\
$20-40$ & 16 & 20.0 & 21.9 & 17.3 \\
$40-60$ & 18 & 14.6 & 16.6 & 16.0 \\
$60-80$ & 13 & 10.6 & 12.0 & 14.5 \\
$80-100$ & 11 & 7.7 & 8.5 & 10.5 \\
$>100$ & 16 & 20.6 & 17.4 & 16.0 \\
\hline & & $\hat{\theta}=0.01588$, & & $X^{2}=0.53, \mathrm{df}=2$, \\
& & $X^{2}=4.61, \mathrm{df}=4$, & $X^{2}=0.64, \mathrm{df}=2$, & $\mathrm{p}$-value 0.77 \\
& & $\mathrm{p}$-value 0.33 & p-value 0.72 \\
& & $V_{2}^{2}=1.54$, & & \\
& & p-value 0.21 & & \\
& & $V_{3}^{2}=2.46$, & & \\
& & p-value 0.12 & & \\
& & &
\end{tabular}

Table 9. Fourth bus-motor failures.

\begin{tabular}{|c|c|c|c|c|}
\hline & & \multicolumn{3}{|c|}{$\begin{array}{c}\text { Expected } \\
\text { frequency }\end{array}$} \\
\hline $\begin{array}{c}\text { Class Interval } \\
(1,000 \text { miles })\end{array}$ & $\begin{array}{c}\text { Observed } \\
\text { frequency }\end{array}$ & Exponential & $\begin{array}{c}\text { Exponentiated } \\
\text { Weibull }\end{array}$ & $n p_{j}^{*}$ \\
\hline $0-20$ & 34 & 34.3 & 34.0 & 34.4 \\
$20-40$ & 20 & 22.1 & 19.5 & 18.3 \\
$40-60$ & 15 & 14.2 & 16.2 & 17.5 \\
$60-80$ & 15 & 9.1 & 14.3 & 13.3 \\
$>80$ & 12 & 16.3 & 12.0 & 12.4 \\
\hline & & $\hat{\theta}=0.02214$, & & $X^{2}=0.74, \mathrm{df}=1$, \\
& & $X^{2}=5.22, \mathrm{df}=3$, & $X^{2}=0.13, \mathrm{df}=1$, & $\mathrm{p}$-value 0.39 \\
& & $\mathrm{p}$-value 0.16 & $\mathrm{p}$-value 0.71 \\
& & $V_{2}^{2}=0.83$, & & \\
& & $\mathrm{p}$-value 0.36 & & \\
& & $V_{3}^{2}=3.49$, & & \\
& & $\mathrm{p}$-value 0.06 & & \\
& & &
\end{tabular}

with the exponentiated Weibull model. We suggest that our analysis adds significant value by indicating what the deviations from the grouped exponential model are, and by providing a simple alternative model which is also applicable to other grouped data, such as grouped normal-like data.

The exponentiated Weibull expected frequencies and the associated $X^{2}$, degrees of freedom (df) and p-values are taken from Mudholkar et al. (1995). Our value of $X^{2}$ for the exponential fit differs from that of Mudholkar et al. (1995) because we use method of moments estimation, not maximum likelihood estimation. All p-values quoted use approximating $\chi^{2}$ distributions even though we have not further grouped the data in the tails as did Mudholkar et al. (1995). 
Table 10. Fifth bus-motor failures.

\begin{tabular}{|c|c|c|c|c|}
\hline & & \multicolumn{3}{|c|}{$\begin{array}{l}\text { Expected } \\
\text { frequency }\end{array}$} \\
\hline $\begin{array}{l}\text { Class Interval } \\
(1,000 \text { miles })\end{array}$ & $\begin{array}{l}\text { Observed } \\
\text { frequency }\end{array}$ & Exponential & $\begin{array}{c}\text { Exponentiated } \\
\text { Weibull }\end{array}$ & $n p_{j}^{*}$ \\
\hline $0-20$ & 29 & 35.0 & 29.0 & 29.2 \\
\hline $20-40$ & 27 & 20.6 & 26.6 & 26.2 \\
\hline $40-60$ & 14 & 12.1 & 14.8 & 15.3 \\
\hline $60-80$ & 8 & 7.1 & 7.4 & 7.2 \\
\hline$>80$ & 7 & 10.2 & 7.1 & 7.2 \\
\hline & & $\begin{array}{c}\hat{\theta}=0.02650, \\
X^{2}=4.42, \mathrm{df}=3, \\
\text { p-value } 0.22 \\
V_{2}^{2}=3.94, \\
\text { p-value } 0.05 \\
V_{3}^{2}=0.21, \\
\text { p-value } 0.65\end{array}$ & $\begin{array}{c}X^{2}=0.09, \mathrm{df}=1, \\
\text { p-value } 0.77\end{array}$ & $\begin{array}{c}X^{2}=0.24, \mathrm{df}=1, \\
\text { p-value } 0.62\end{array}$ \\
\hline
\end{tabular}

For the first data set the fit from $\left\{n p_{j}^{*}\right\}$ is not quite as good as for the exponentiated Weibull model, but an acceptable p-value of 0.20 is obtained. More accurate p-values could be found via parametric bootstrap, but these would not change the inference. Including extra $V_{r}$ 's in $p_{j}^{*}$ might improve the fit, but we did not consider that here. Similar comments apply to the second and third data sets, except that for the latter the fit from $\left\{n p_{j}^{*}\right\}$ is slightly better than for the exponentiated Weibull model: see Table 8. Note that here we improve on the exponential model even though that model is consistent with the data: none of $S W 2, V_{2}^{2}$ and $V_{3}^{2}$ are significant.

For the fourth bus motor failure data the SW2 test applied by Gulati and Neus (2003) had p-value 0.33 , but we find $V_{3}^{2}$ has p-value 0.06 , casting doubt on the null exponential model. Once again we can use $V_{2}$ and $V_{3}$ to give an improved model comparable with the exponentiated Weibull.

For the final data set Gulati and Neus (2003) found $S W 2=2.34$ with p-value 0.04, and conclude the null exponential model is in doubt. We can say more. Our components of $X^{2}$ show that the data are likely to be underdispersed $\left(V_{2}=-1.985\right)$ compared to the exponential, and our expected values based on adjusting the exponential model using $V_{2}$ and $V_{3}$ again gives an improved model. A model using just $V_{2}$ would give a similar fit but a larger p-value, similar to that for the exponentiated Weibull model. Notice also that for this final data set $V_{2}^{2}$ is significant while $X^{2}$ is not, and we have an example of the dilution effect mentioned in section 2 above.

\section{CONCLUSION}

We recommend testing for a grouped exponential model using one of the statistics $A^{2}$ or $S W 2$. Further, this use should be complemented by examining $X^{2}$ and its first two non-zero components $V_{2}^{2}$ and $V_{3}^{2}$. The former has approximate $\chi_{K-2}^{2}$ distribution and the latter $\chi_{1}^{2}$ when the MME and MLE are close and the class expectations are not too small. The parametric bootstrap can be used to calculate both p-values and powers. When all class expectations are greater than one and mostly greater than five, the chi-squared approximation 
can be used to estimate p-values and the non-central chi-squared approximation can be used to estimate powers for the tests based on $V_{2}^{2}, V_{3}^{2}$ and $X^{2}$. When the null hypothesis is rejected improved models can be constructed by making adjustments corresponding to the components with sufficiently small p-values. These points have been illustrated using data related to river bed scouring and bus motor failure.

\section{REFERENCES}

Best, D.J. and Rayner, J.C.W. (2003). Tests of fit for the geometric distribution. Communications in Statistics, Simulation and Computation, 32 (4), 1065 - 1078.

Best, D.J. and Rayner, J.C.W. (2005). Improved testing for the Poisson distribution using chisquared components with data dependent cells. Communications in Statistics, Simulation and Computation, 34 (1), 85 - 96.

Best, D.J. and Rayner, J.C.W. (2006). Improved testing for the binomial distribution using chisquared components with data dependent cells. Journal of Statistical Computation and Simulation, 76, 75-81.

Davis, D.J. (1952). An analysis of some failure rate data. Journal of the American Statistical Association, 47, 113 - 150.

Emerson, P.L. (1968). Numerical construction of orthogonal polynomials from a general recurrence formula. Biometrics, 24, 695 - 701.

Gulati, S. and Neus, J. (2001). Goodness-of-fit statistics for the exponential distribution when the data are grouped. Chapter 9 in Goodness-of-Fit Tests and Validity of Models, eds. Huber-Carol, C., Balakrishnan, N., Nikulin, M. S. and Mesbah, M., Boston: Birkhauser, $113-123$.

Gulati, S. and Neus, J. (2003). Goodness of fit statistics for the exponential distribution when the data are grouped. Commun. Statist.-Theor. Meth., 32, $681-700$.

IMSL (1995). Users' Manual. Houston: IMSL.

Lancaster, H.O. (1953). A reconciliation of $\chi^{2}$ from metrical and enumerative aspects. Sankhya, 13, $1-10$.

Mudholkar, G.S., Srivastava, D.K. and Freimer, M. (1995). The exponential Weibull family: a reanalysis of the bus-motor failure data. Technometrics, 37, 436 - 445.

Rayner, J.C.W. and Best, D.J. (1989). Smooth Tests of Goodness of Fit. New York: Oxford University Press.

Rayner, J.C.W., Best, D.J. and Mathews, K.L. (1995). Interpreting the skewness coefficient. Commun. Statist.-Theor. Meth., 24(3), 593 - 600.

Stuart, A. and Ord, J.K. (1994). Kendall's Advanced Theory of Statistics, Vol 1 (6 $6^{\text {th }}$ ed.). London: Edward Arnold.

Thas, O., Rayner, J.C.W., and Best, D.J. (2006). Smooth tests for the logistic distribution. Submitted.

Spinelli, J.J. (2001). Testing for fit for the grouped exponential distribution. Canadian Journal of Statistics, 29, 451 - 458. 